\title{
Carotid Bulb Webs as a Cause of "Cryptogenic" Ischemic Stroke
}

\author{
DP.I. Sajedi, (D).N. Gonzalez, (D) C.A. Cronin, (D)T. Kouo, (D)A. Steven, (D). Zhuo, (D). Thompson, (D) R. Castellani, (D).J. Kittner, \\ (DD. Gandhi, and (DP. Raghavan
}

\begin{abstract}
BACKGROUND AND PURPOSE: Carotid webs are intraluminal shelf-like filling defects at the carotid bulb with recently recognized implications in patients with recurrent ischemic stroke. We sought to determine whether carotid webs are an under-recognized cause of "cryptogenic" ischemic stroke and to estimate their prevalence in the general population.
\end{abstract}

MATERIALS AND METHODS: A retrospective review of neck CTA studies in young patients with cryptogenic stroke over the past 6 years $(n=33)$ was performed to determine the prevalence of carotid webs compared with a control group of patients who received neck CTA studies for reasons other than ischemic stroke $(n=63)$.

RESULTS: The prevalence of carotid webs in the cryptogenic stroke population was $21.2 \%(95 \% \mathrm{Cl}, 8.9 \%-38.9 \%)$. Patients with symptomatic carotid webs had a mean age of 38.9 years (range, $30-48$ years) and were mostly African American (86\%) and women (86\%). In contrast, only $1.6 \%(95 \% \mathrm{Cl}, 0 \%-8.5 \%)$ of patients in the control group demonstrated a web. Our findings demonstrate a statistically significant association between carotid webs and ischemic stroke (OR $=16.7 ; 95 \% \mathrm{Cl}, 2.78-320.3 ; P=.01)$.

CONCLUSIONS: Carotid webs exhibit a strong association with ischemic stroke, and their presence should be suspected in patients lacking other risk factors, particularly African American women.

C arotid artery webs are shelf-like intraluminal protrusions in the carotid bulb with emerging implications related to recurrent ischemic stroke. ${ }^{1,2}$ Most carotid web cases have been previously described with conventional angiography. ${ }^{3,4}$ More recently, the imaging characteristics on CTA have also been established. The typical appearance of a carotid web on CTA is a focal, gracile intraluminal filling defect along the posterior wall of the carotid bulb. ${ }^{1,5}$ Superimposed thrombus has also been described, which is thought to be related to sluggish/turbulent blood flow produced by the filling defect. ${ }^{5}$

Carotid webs also have been referred to as an atypical variant of fibromuscular dysplasia, with intimal fibrosis and hyperplasia on histology in contrast to the classic, medial variant. ${ }^{3,6}$ Typical fibromuscular dysplasia occurs in middle-aged white women,

Received December 26, 2016; accepted after revision February 27, 2017

From the Departments of Diagnostic Radiology and Nuclear Medicine (P.R., P.I.S. T.K., J.Z.), Neurology (J.N.G., C.A.C., O.T., S.J.K.), and Interventional Neuroradiology (D.G.), University of Maryland School of Medicine, Baltimore, Maryland; Department of Diagnostic Radiology and Nuclear Medicine (A.S.), Ochsner Medical Center, New Orleans, Louisiana; and Department of Pathology (R.C.), University Homer Stryker M.D. School of Medicine, Kalamazoo, Michigan.

Please address correspondence to Prashant Raghavan, MBBS, Department of Diagnostic Radiology and Nuclear Medicine, University of Maryland School of Medicine, 22 South Greene St, Baltimore, MD 21201; e-mail: prashant.raghavan@gmail.com

http://dx.doi.org/10.3174/ajnr.A5208 with a classic "string of beads" imaging appearance, and does not have a direct association with ischemic stroke. ${ }^{6,7}$

Although considered a rare entity, a significant proportion of reported carotid web cases have been associated with recurrent ischemic strokes, most frequently in younger adults who lack other known risk factors. ${ }^{1}$ Recent studies have revealed a mean age between 45 and 50 years in patients with carotid webs and associated ipsilateral carotid territory ischemic strokes, occurring more frequently in women than men. ${ }^{1,5,8}$ There is limited reporting on the prevalence of carotid webs in the stroke population. A recent report on an Afro-Caribbean population demonstrated a $23 \%$ prevalence of carotid webs in young patients with ischemic stroke and a $7 \%$ prevalence among control patients. ${ }^{8}$ Up to one-third of all patients presenting with ischemic strokes lack an identifiable cause and are classified as "cryptogenic" in etiology, with most of these cases occurring in younger patients. ${ }^{9}$ Webs may be an under-recognized entity because of their subtle morphology and a lack of familiarity amongst radiologists and clinicians with this lesion. They could account for a significant portion of cryptogenic strokes, particularly in young adults.

The purpose of our study was to determine the prevalence of carotid webs in a group of patients previously classified as having cryptogenic stroke. 


\section{MATERIALS AND METHODS}

\section{Patient Selection: Cryptogenic Stroke Group}

This retrospective study was approved by our institutional review board; the need for informed consent was waived. A chart review of all patients presenting to the University of Maryland Medical Center with ischemic strokes from January 1, 2010 to August 31, 2015 ( $n=1738$ ) was undertaken. Patients between 18 and 55 years of age $(n=590)$ were examined in detail to identify those with anterior circulation cryptogenic stroke. Stroke etiology determination was modeled after the Causative Classification System, with patients characterized into one of the following categories after thorough work-up: large artery atherosclerotic, cardio-aortic embolism, small artery occlusion, other known cause, or cryptogenic. ${ }^{10}$ The work-up for these patients was as follows: all patients received transthoracic echocardiogram, with transesophageal echocardiogram and evaluation for right-to-left shunt performed if the etiology remained unknown after the initial evaluation. All patients had cardiac telemetry monitoring for arrhythmia, with prolonged ambulatory monitoring when indicated. Intracranial vascular imaging was performed with CTA or MRA, and extracranial vascular imaging was performed with CTA, MRA, or carotid duplex sonography. The potential for underlying dissection as the etiology was excluded by history and imaging. Lumbar puncture and laboratory testing to evaluate for appropriate etiologies, such as vasculitis, other autoimmune disorders, or hypercoagulable state, were performed when clinically indicated. A total of 119 patients between 18 and 55 years old with anterior circulation cryptogenic stroke were identified.

Forty of 119 patients with cryptogenic stroke had a diagnostic neck CTA performed during their admission and were included in the analysis. These neck CTA studies were transferred to the Aquarius Workstation (version 3.6.2.3; TeraRecon, San Mateo, California), an independent imaging viewer that enables multiplanar reformation as well as MIPs. Studies were then evaluated by a board-certified, fellowship-trained neuroradiologist with more than 10 years of experience to exclude patients with atherosclerotic calcification and/or greater than 3-mm carotid wall plaque within $2 \mathrm{~cm}$ above and below the level of the carotid bulb. This criteria was assigned because of recent studies that have shown that nonstenotic atherosclerotic disease above this threshold may account for cases of cryptogenic ischemic stroke. ${ }^{1-13}$ Seven cases of atherosclerosis that met this criteria were identified by the reader, with 1 additional study deemed nondiagnostic because of inadequate contrast bolus timing, leaving a total of 33 neck CTA studies in our case population (Fig 1).

\section{Patient Selection: Control Group}

The control group was composed of patients between 18 and 55 years of age who had CTA of the neck performed during their admission and who presented for reasons other than ischemic stroke (including trauma, presurgical planning, etc). We excluded any CTA studies that demonstrated direct trauma to the neck (blunt or penetrating) or studies demonstrating a cervical spine fracture. Furthermore, patients with intracranial vascular lesions, including aneurysms and AVMs, were also excluded from the study because of a link between intracranial aneurysms and fibromuscular dysplasia. ${ }^{7}$ The control group was chosen to be compa-

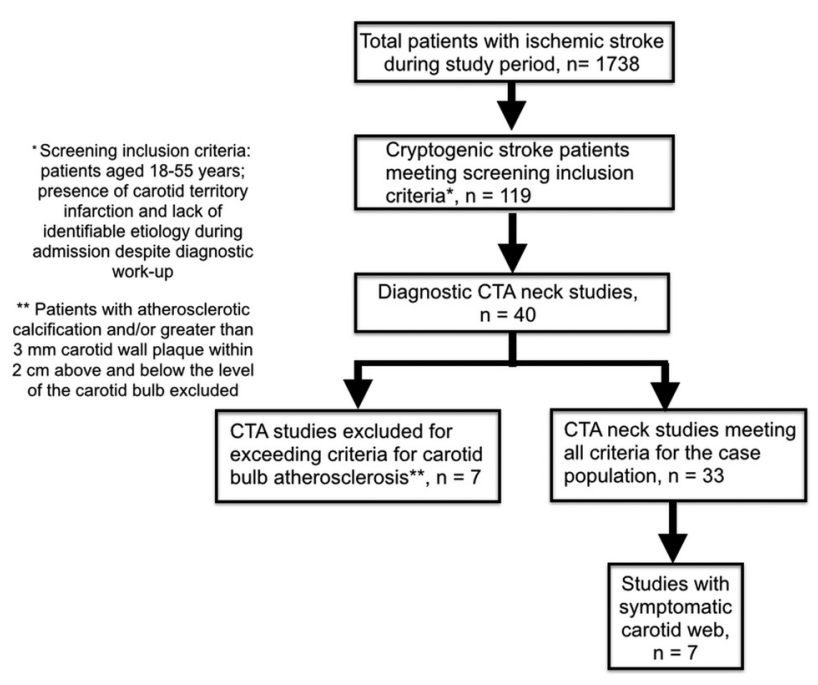

FIG 1. Algorithm of inclusion criteria.

Table 1: Demographic features of patients with cryptogenic stroke and control group patients

\begin{tabular}{lccc}
\hline \multicolumn{1}{c}{ Characteristic } & $\begin{array}{c}\text { Patients with } \\
\text { Cryptogenic } \\
\text { Stroke }(\boldsymbol{n}=33)\end{array}$ & $\begin{array}{c}\text { Control Group } \\
\text { Patients } \\
(\boldsymbol{n}=63)\end{array}$ & $\begin{array}{c}\boldsymbol{P} \\
\text { Value }\end{array}$ \\
\hline Age, mean, SD & $39.2,8.3$ & $39.6,8.4$ & .82 \\
Sex, no. (\%) & $11(33)$ & $21(33)$ & .83 \\
$\quad$ Male & $22(67)$ & $42(67)$ & \\
$\quad$ Female & $18(54)$ & $34(54)$ & .89 \\
Ethnicity, no. (\%) & $14(43)$ & $28(44)$ & \\
$\quad$ African American & $1(3)$ & $1(2)$ & \\
$\quad$ White & $7(21.2)$ & $1(1.6)$ & .002 \\
$\quad$ Other & & & \\
Carotid webs, no. (\%) & & & \\
\hline
\end{tabular}

rable with the case group with regard to age, sex, and ethnicity (Table 1). A total of 63 patients who met the inclusion criteria and had neck CTA studies done were acquired between November 6 , 2012 and August 31, 2015. The following factors were assessed: patient age, sex, and indication for the CTA. As with the neck CTA studies in patients with ischemic stroke, these studies were transferred to the Aquarius Workstation.

\section{Imaging Evaluation}

CTA was chosen as the technique for interpretation because of its reliability in characterizing carotid webs ${ }^{1,5,8}$ as well as its ability to identify alternate pathologies such as atherosclerosis and vascular injury. ${ }^{5}$ Neck CTA was acquired from the aortic arch through the circle of Willis and performed on a 64-MDCT scanner with 1-mm thick sections. Scanning parameters included: pitch of 1 ; gantry rotation time, 0.5 seconds; $120 \mathrm{kVp} ; 350 \mathrm{mAs}$. Intravenous contrast material $(100 \mathrm{~mL}$ of iohexol, $300 \mathrm{mg} / \mathrm{mL}$; Omnipaque, GE Healthcare, Piscataway, New Jersey) and a 50-mL saline flush were administered at a rate of $4 \mathrm{~mL} / \mathrm{s}$ with a power injector. Images were reconstructed with axial, coronal, and sagittal MIPs.

An independent review of these studies was then performed by 2 board-certified, fellowship-trained neuroradiologists with a range of 6-8 years of experience (T.K. and A.S.). The readers were blinded to patient age, sex, and laterality of stroke. The following parameters were assessed by each reader: presence of carotid web, 

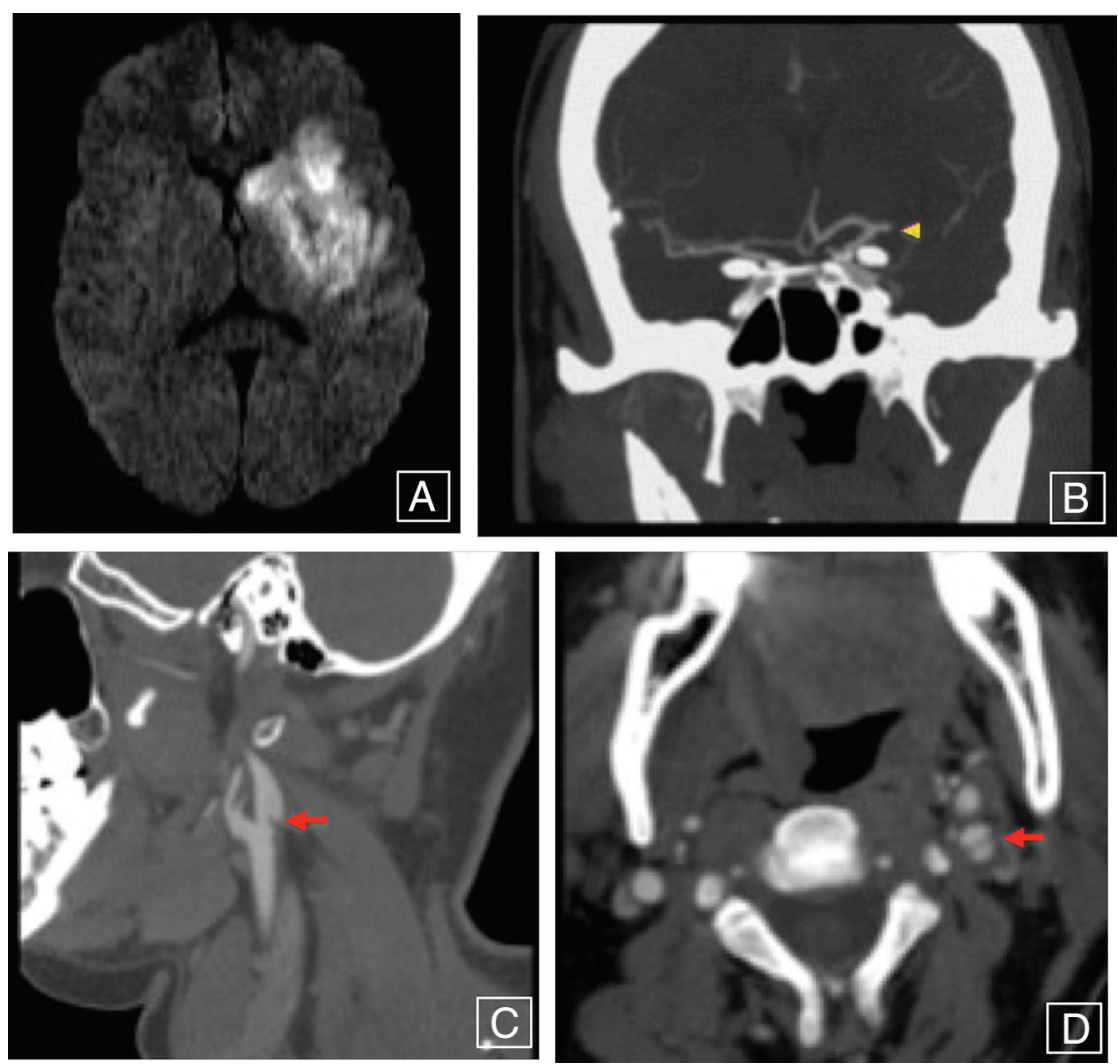

FIG 2. A patient presenting with right-sided weakness demonstrating an acute, large left MCA territory infarct as seen on diffusion-weighted imaging $(A)$, with coronal CTA MIP image $(B)$ showing abrupt occlusion involving the M1 segment of the left MCA (yellow arrowhead). Sagittal and axial neck CTA images $(C$ and $D)$ reveal a shelf-like intraluminal protrusion arising from the posterior wall of the left carotid bulb (red arrows), compatible with a carotid web. bulb atherosclerotic calcifications or wall thickness greater than $3 \mathrm{~mm} .^{11-13}$

Both readers agreed on the presence and laterality of $6(18.2 \%)$ cases of carotid web (Cohen $\kappa$ coefficient, 0.78 ; 95\% CI, 0.55-1) (Fig 2). Carotid webs were present bilaterally in half of these patients. Disagreement was encountered in 2 patients. Upon review of the studies by the third reader, 1 additional patient was deemed to have a web. Thus, a total of $7(21.2 \%)$ positive cases of carotid web were ultimately identified within the cryptogenic stroke population (Table 2). One patient with a web demonstrated superimposed clot formation. No patients with coexistent typical, medial variant fibromuscular dysplasia were encountered.

Among the cases that were negative for carotid web, both readers agreed on 1 $(3.8 \%)$ case with classic features of typical fibromuscular dysplasia. Features of prior carotid vascular injury, including pseudoaneurysm and dissection, were seen in $2(7.7 \%)$ patients, though contralateral to the stroke. A thorough review of each patient's clinical presentation did not demonstrate a history suggestive of recent cervical vascular injury, such as neck pain, recent neck manipulation, hyperextension, or trauma.

Of the 63 patients in the control group, both readers agreed on $1(1.6 \%)$ patient with incidental findings of carotid web. This patient was a 48-year-old African American woman whose imaging demonstrated no evidence of superimposed clot, atherosclerosis, or features of classic fibromuscular dysplasia. The prevalences of carotid webs in the cryptogenic stroke and control groups were $21.2 \%$ (CI, 8.9\%-38.9\%) and $1.6 \%$ (CI, 0\%-8.5\%), respectively, with a statistically significant association between webs and ischemic stroke $(\mathrm{OR}=16.7 ; 95 \% \mathrm{CI}, 2.78-320.3 ; P=$ $.01)$. The mean age of patients with symptomatic carotid webs was 38.3 years (range, $30-41$ years) compared with 42.5 years in patients with cryptogenic stroke without a carotid web (Table 3 ). The symptomatic patients with carotid web were 6 (86\%) women and $1(14 \%)$ man, and the remainder of the cryptogenic stroke population, those without carotid webs, consisted of $16(62 \%)$ women and $10(38 \%)$ men. With regard to ethnicity, $6(86 \%)$ patients with carotid web and stroke were African American and 1 (14\%) was white. In contrast, the ethnic makeup of the patients with noncarotid web included 12 African Americans (46\%), 13 whites (50\%), and 1 Asian (4\%). There was no statistically significant difference in risk factors for patients with stroke such as hypertension; dyslipidemia; diabetes mellitus; and alcohol, cocaine, and tobacco use between the 2 groups (Table 3 ).

All symptomatic patients with carotid webs demonstrated MCA territory infarction ipsilateral to the carotid web. These in-

\section{RESULTS}

Of the 119 patients between the ages of 18 and 55 years who presented to our institution and were diagnosed with cryptogenic stroke during the study time period, 33 (27.7\%) underwent a diagnostic neck CTA after accounting for patients with carotid 
Table 2: Detailed demographic and imaging/clinical features, including work-up, of patients with positive carotid webs in the cryptogenic stroke population ${ }^{\mathrm{a}}$

\begin{tabular}{|c|c|c|c|c|c|c|c|}
\hline $\begin{array}{l}\text { Patient } \\
\text { No. }\end{array}$ & Ethnicity & Stroke Location & $\begin{array}{l}\text { Thrombus } \\
\text { Location }\end{array}$ & $\begin{array}{c}\text { Web } \\
\text { Location }\end{array}$ & $\begin{array}{l}\text { Work-Up } \\
\text { Methods }\end{array}$ & $\begin{array}{c}\text { Management } \\
\text { after Initial Event }\end{array}$ & $\begin{array}{l}\text { Recurrent } \\
\text { Stroke }\end{array}$ \\
\hline 1 & White & $\begin{array}{l}\text { Near complete right MCA } \\
\text { territory infarction }\end{array}$ & Right Ml & Bilateral & $1-3^{c}$ & Supportive care & No \\
\hline 2 & African American & $\begin{array}{l}\text { Near complete right MCA } \\
\text { territory infarction }\end{array}$ & Right M1 & Right & $1-3^{c}$ & Clot retrieval & No \\
\hline 3 & African American & $\begin{array}{l}\text { Right basal ganglia and } \\
\text { temporal lobe infarcts }\end{array}$ & $\begin{array}{l}\text { Right distal M1/ } \\
\text { proximal M2 }\end{array}$ & Right & $1-3$ & Supportive care & No \\
\hline 4 & African American & $\begin{array}{l}\text { Near complete left MCA } \\
\text { territory infarction }\end{array}$ & $\begin{array}{l}\text { Left distal M1/ } \\
\text { proximal M2 }\end{array}$ & Bilateral & $1-3$ & Supportive care & $\begin{array}{l}\text { Yes, multiple } \\
\text { recurrent }\end{array}$ \\
\hline 5 & African American & $\begin{array}{l}\text { Near complete left MCA } \\
\text { territory infarction }\end{array}$ & Left M1 & Bilateral & $1-3^{c}$ & $\begin{array}{l}\text { Clot retrieval, then } \\
\text { CEA }\end{array}$ & $\mathrm{N} / \mathrm{A}$ \\
\hline 6 & African American & $\begin{array}{l}\text { Left basal ganglia and } \\
\text { insular cortex infarcts }\end{array}$ & Left M2 & Left & $1-3$ & $\begin{array}{l}\text { Supportive care, } \\
\text { then CEA }\end{array}$ & $\mathrm{N} / \mathrm{A}$ \\
\hline 7 & African American & $\begin{array}{l}\text { Left basal ganglia and } \\
\text { insular cortex infarcts }\end{array}$ & Left M2 & Left & $1-3$ & Supportive care & No \\
\hline
\end{tabular}

Note:-CEA indicates carotid endarterectomy; M1, segment from the origin to the bifurcation/trifurcation of the MCA; M2, segment from the MCA bifurcation/trifurcation to the insular circular sulcus; N/A, no follow-up available.

a Patient ages ranged from 30-48 years, composed of 6 women and 1 man.

b Work-up of patients with carotid web: 1, echocardiogram; 2, cardiac telemetry; 3, cross-sectional head/neck vascular imaging.

c Interventional head/neck angiogram also performed.

Table 3: Demographic features and pertinent risk factors of patients with and without carotid webs in the cryptogenic stroke population $^{\mathrm{a}}$

\begin{tabular}{lccc}
\hline & $\begin{array}{c}\text { Patients with } \\
\text { Cryptogenic } \\
\text { Stroke with } \\
\text { Carotid Web } \\
(\boldsymbol{n}=7)\end{array}$ & $\begin{array}{c}\text { Patients with } \\
\text { Cryptogenic } \\
\text { Stroke without } \\
\text { Carotid Web } \\
(\boldsymbol{n}=26)\end{array}$ & $\begin{array}{c}\boldsymbol{P} \\
\text { Value }\end{array}$ \\
\hline Characteristics & $38.3,5.6$ & $39.4,9.0$ & .721 \\
\hline $\begin{array}{l}\text { Age, mean, SD } \\
\text { Sex, no. (\%) }\end{array}$ & $1(14)$ & $10(38)$ & .38 \\
Male & $6(86)$ & $16(62)$ & \\
Female & & $12(46)$ & .16 \\
Ethnicity, no. (\%) & $6(86)$ & $13(50)$ & \\
African American & $1(14)$ & $1(4)$ & \\
White & $0(0)$ & $8(31)$ & .15 \\
Other & $0(0)$ & $4(15)$ & .58 \\
Risk factors, no. (\%) & $2(29)$ & $7(27)$ & .30 \\
Hypertension & $0(0)$ & $5(19)$ & .56 \\
Dyslipidemia & $0(0)$ & $0(0)$ & N/A \\
Diabetes mellitus & $0(0)$ & $6(23)$ & .39 \\
Alcohol abuse & $3(43)$ & & \\
Cocaine & &
\end{tabular}

Note:- N/A indicates not available.

${ }^{a}$ Added $P$ values for patient characteristics.

farcts ranged in size from involvement of the basal ganglia and insular cortex to involvement of greater than two-thirds of the MCA territory (Table 2). None of these patients had the appearance of a lacunar infarct. Of these 7 symptomatic patients with carotid web, $1(14.3 \%)$ had multiple documented episodes of recurrent stroke. This patient was treated with intravenous tPA during each encounter, with the family refusing surgical intervention with carotid endarterectomy. In the remaining 6 patients, 4 were treated with supportive care during the initial episode and 2 with mechanical thrombectomy. After the presence of web was identified, 2 patients underwent carotid endarterectomy.

Seventy-eight patients were excluded from the case group given the lack of neck CTA imaging at presentation. Still, each of these patients did receive cervical vascular imaging in the form of MRA and/or carotid duplex sonography to rule out standard etiologies of stroke. An imaging review of these patients, performed in retrospect, showed no patients with definite features of carotid web.

\section{DISCUSSION}

Our findings reveal a statistically significant association between carotid webs and ischemic stroke, particularly in younger African American women, with a prevalence of $21.2 \%$ in the cryptogenic stroke population as opposed to $1.6 \%$ in the control group, yielding an odds ratio of 10.6 (95\% CI, 1.8-203.1). By comparison, a smaller case-control analysis of cryptogenic stroke in the AfroCaribbean population of Martinique in the French West Indies found a prevalence of $37 \%$ in their 23 patients with cryptogenic stroke and a prevalence of $7 \%$ in their control patients, yielding an odds ratio of 5.0 (95\% CI, 1.1-22.8). ${ }^{8}$ A more recent case-control analysis by Coutinho et $\mathrm{al}^{11}$ also revealed similar findings, albeit with a lower prevalence of webs in cases $(9.4 \%)$ compared with control patients $(1 \%$; OR $=8.0 ; 95 \% \mathrm{CI}, 1.2-67 ; P=.032) \mathrm{com}-$ pared with our series. They also noted a lack of significant difference in the prevalence of nonstenotic atherosclerotic disease between the case and control groups.

In our study, all patients with stroke who had carotid webs had them ipsilateral to the stroke; 3 had bilateral webs. All patients in this group had middle cerebral territory (MCA) infarcts, 5 (71.4\%) with large territory involvement. These findings support our hypothesis that carotid webs are found more frequently in patients with cryptogenic strokes than in control patients and are likely causative. Our findings are in concordance with the emerging literature on this topic, with a higher prevalence of carotid webs in African American female patients with ischemic stroke who are of younger age and lack other identifiable risk factors. ${ }^{1,5,8}$ It should be noted that our 7 cases of cryptogenic stroke with carotid web included only 1 man and 1 person of white race. The mechanism of stroke in such patients, as suggested by Choi et al, ${ }^{5}$ may be related to local turbulence and subsequent thromboembolism. This is supported by the presence of a coexistent thrombus in 1 patient in our series.

CTA was used as the technique of choice in our study. Al- 

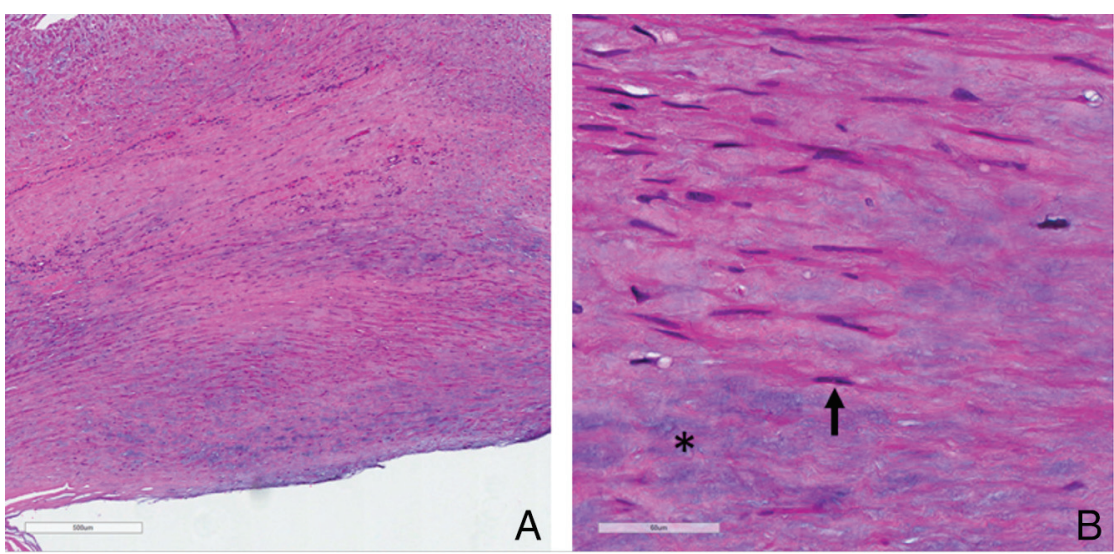

FIG 3. Low-magnification photomicrograph $(A)$ shows extensive fibromuscular proliferation (scale bar $=500 \mu$ ). High magnification $(B)$ shows proliferating smooth muscle cells (arrow) and myxoid degeneration (asterisk).

though descriptions of webs in conventional angiography, MR angiography, and sonography exist in the literature, CTA appears to be reliable in the detection and characterization of this entity. ${ }^{14,15}$ The advantages of CTA include rapid, high-resolution imaging as well as the ability to obtain reformatted images in multiple planes. Furthermore, CTA provides the added benefit of characterizing superimposed findings, such as thrombus, and aids in the exclusion of atherosclerosis or vascular injury. In the group of 78 patients with cryptogenic strokes who did not undergo CTA, no webs were found. This may have been because of limitations imposed by operator dependence (ultrasound), technique (TOF MRA), and artifacts. Therefore, based on our experiences, we suggest that all patients with cryptogenic stroke receive CTA imaging of the neck as part of the standard work-up.

Carotid webs can be distinguished from dissection and atherosclerotic plaques by their characteristic location and appearance, being gracile and focal in nature and exclusively located in the posterior wall of the carotid bulb. In contrast, dissection flaps typically propagate beyond the carotid bulb and may be associated with intramural hematomas and/or pseudoaneurysms. Soft carotid plaques present as focal areas of mural thickening, often, but not necessarily, confined to the posterior wall of the carotid bulb.

The prospective diagnosis of carotid webs will allow for targeted intervention to prevent recurrent ischemic events. Although 2 of our patients underwent carotid endarterectomy, there may also be a role for endovascular stent placement in the management of carotid webs, as described by Elmokadem et al. ${ }^{16}$ Histopathology in the patients who underwent surgery showed extensive intimal fibromuscular proliferation with fibrosis and myxoid degeneration. No frank atheromatous change was seen in either patient. These findings were most consistent with an atypical, intimal variant form of fibromuscular dysplasia, in keeping with prior descriptions of the histopathologic features of this entity (Fig 3). ${ }^{5}$

Our study had several potential limitations, including a retrospective study design and a small sample size. In addition, we included only patients between 18 and 55 years old, and it is conceivable that webs may be encountered in patients younger and older than our cohort. In fact, we have recently identified at least
2 patients with ischemic stroke with imaging features of carotid web who were older than 55 years. Another potential limitation of our study was evaluating only patients who had a CTA of the neck performed. Seventy-eight young patients with cryptogenic stroke were not included within the study population secondary to a lack of neck CTA imaging as detailed above. In addition, histopathologic correlation was available for only 2 patients in our series, though 1 patient's family refused surgical intervention, and several of the remaining cases had large territory infarcts, potentially limiting any benefit of an invasive procedure.

\section{CONCLUSIONS}

Carotid webs are an under-recognized cause of ischemic anterior circulation stroke, especially in younger African American women. CT angiography reliably depicts webs as gracile crescentic filling defects arising from the posterior wall of the carotid bulb and should be considered as part of the evaluation for patients with cryptogenic stroke. Histopathologically, they represent an intimal variant of fibromuscular dysplasia. Definitive treatment may require carotid endarterectomy or endovascular stent placement.

Disclosures: Steven Kittner-UNRELATED: Employment: University of Maryland, Veterans Administration; Grants/Grants Pending: National Institutes of Health, Veterans Administration*; Payment for Lectures (including service on Speakers Bureaus): National Institutes of Health. Dheeraj Gandhi-UNRELATED: Royalties: Cambridge Press. *Money paid to the institution.

\section{REFERENCES}

1. Joux J, Chausson N, Jeannin S, et al. Carotid-bulb atypical fibromuscular dysplasia in young Afro-Caribbean patients with stroke. Stroke 2014;45:3711-13 CrossRef Medline

2. Morgenlander JC, Goldstein LB. Recurrent transient ischemic attacks and stroke in association with an internal carotid artery web. Stroke 1991;22:94-98 CrossRef Medline

3. Wirth FP, Miller WA, Russell AP. Atypical fibromuscular hyperplasia. Report of two cases. J Neurosurgery 1981;54:685-89 CrossRef Medline

4. So EL, Toole JF, Moody DM, et al. Cerebral embolism from septal fibromuscular dysplasia of the common carotid artery. Ann Neurol 1979;6:75-78 CrossRef Medline

5. Choi PM, Singh D, Trivedi A, et al. Carotid webs and recurrent ischemic strokes in the era of CT angiography. AJNR Am J Neuroradiol 2015;36:2134-39 CrossRef Medline

6. Watanabe S, Tanaka K, Nakayama T, et al. Fibromuscular dysplasia at the internal carotid origin: a case of carotid web [in Japanese]. No Shinkei Geka 1993;21:449-52 Medline

7. Touzé E, Oppenheim C, Trystram D, et al. Fibromuscular dysplasia of cervical and intracranial arteries. Int J 2010;5:296-305 CrossRef Medline

8. Joux J, Boulanger M, Jeannin S, et al. Association between carotid bulb diaphragm and ischemic stroke in young Afro-Caribbean patients: a population-based case-control study. Stroke 2016;47: 2641-44 CrossRef Medline

9. Finsterer J. Management of cryptogenic stroke. Acta Neurol Belg 2010;110:135-47 Medline

AJNR Am J Neuroradiol 38:1399-404 Jul 2017 www.ajnr.org 
10. Arsava EM, Ballabio E, Benner T, et al. The Causative Classification of Stroke system: an international reliability and optimization study. Neurology 2010;75:1277-84 CrossRef Medline

11. Coutinho JM, Derkatch S, Potvin AR, et al. Carotid artery web and ischemic stroke: a case-control study. Neurology 2017;88:65-69 CrossRef Medline

12. Gupta A, Baradaran H, Kamel H, et al. Evaluation of computed tomography angiography plaque thickness measurements in high-grade carotid artery stenosis. Stroke 2014;45:740-45 CrossRef Medline

13. Coutinho JM, Derkatch S, Potvin AR, et al. Nonstenotic carotid plaque on CT angiography in patients with cryptogenic stroke. Neurology 2016;87:665-72 CrossRef Medline

14. Lantos JE, Chazen JL, Gupta A. Carotid web: appearance at MR angiography. AJNR Am J Neuroradiol 2016;37:E5-E6 CrossRef Medline

15. Fu W, Crockett A, Low G, et al. Internal carotid artery web: Doppler ultrasound with CT angiography correlation. J Radiol Case Rep 2015;9:1-6 CrossRef Medline

16. Elmokadem AH, Ansari SA, Sangha R, et al. Neurointerventional management of carotid webs associated with recurrent and acute cerebral ischemic syndromes. Interv Neuroradiol 2016;22:432-37 CrossRef Medline 